\section{Clinical Oncology Society of Australia position statement on cancer survivorship care}

CPD

Janette L Vardy, Raymond J Chan, Bogda Koczwara, Karolina Lisy, Richard J Cohn, David Joske, Haryana M Dhillon, Michael Jefford

\section{Background}

Cancer survivors often experience long-term negative consequences of their cancer and cancer treatment. With increasing numbers of survivors and duration of survival, a sustainable model of care is required to better meet the needs of cancer survivors.

\section{Objective}

The aim of this article is to outline the Clinical Oncology Society of Australia Model of Survivorship Care, summarising the critical components of cancer survivorship care.

\section{Discussion}

Recommendations include: a systematic, multidisciplinary care approach that optimises self-management and enhances coordinated and integrated survivor-centred care from diagnosis; stratified care pathways based on survivors' needs, capacity to self-manage and anticipated treatment sequelae; a focus of care on wellness, healthy lifestyle, symptom management and prevention of life-altering and life-threatening late effects in addition to cancer surveillance; development of a treatment summary and care plan; and equitable, timely access to services, while minimising unnecessary use of healthcare services.
WITH ADVANCES IN CANCER SCREENING, detection and treatment, the number of people surviving cancer is increasing rapidly. In 2018, an estimated 140,000 new cases of cancer were diagnosed in Australia, with 1.1 million people having a personal history of cancer. This is expected to increase to 1.9 million by $2040 .{ }^{1}$ In its broadest definition, a person is a cancer survivor from diagnosis for the remainder of their life. ${ }^{2}$

Cancer survivors often experience long-term negative consequences of their cancer and cancer treatment, in addition to the risk of a cancer recurrence or a second primary cancer. Cancer survivors have an increased risk of cardiovascular disease, type 2 diabetes, metabolic syndrome and osteoporosis. ${ }^{2-6}$ Many have long-term residual symptoms, with more than half of early-stage survivors reporting five or more symptoms of at least moderate severity 12 months after diagnosis, ${ }^{7}$ and fatigue, loss of strength, pain, sleep disturbance and weight changes up to five years after diagnosis. ${ }^{8,9}$ Cognitive impairment, fatigue and other symptoms can affect independent functional ability and return to work, decreasing financial security. ${ }^{10,11}$ Other common physical long-term treatment effects include sexual dysfunction, infertility and chemotherapy-induced peripheral neuropathy. ${ }^{12}$ Late-term effects include second malignancies, and impaired cardiac and pulmonary function. ${ }^{12}$

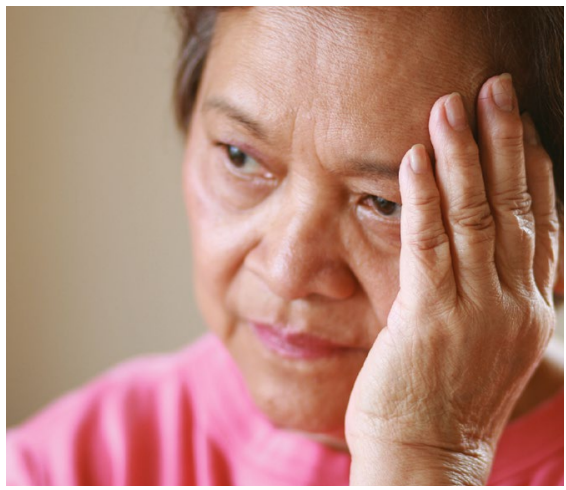

Fear of cancer recurrence occurs in approximately $70 \%$ of survivors, with approximately $50 \%$ reporting fear of at least moderate severity, ${ }^{13}$ high levels of uncertainty about the future, ${ }^{8}$ and unmet needs focused on fear of relapse. ${ }^{14}$ Changes in social roles, support networks and family and intimate relationships often occur, creating added distress. ${ }^{15}$

To address these unique needs of cancer survivors, there have been a number of recommendations for delivery of survivorship care. The seminal report From cancer patient to cancer survivor: Lost in transition defined essential components of survivorship care. ${ }^{16}$ These included prevention of recurrent and new cancers and late effects of treatment; surveillance for cancer spread, recurrence, second cancers, and physical and psychosocial late effects; interventions for the consequences of cancer and its treatment; and coordination between the specialist cancer care team and primary care providers to ensure survivors' health needs are met.

In 2017, Cancer Australia released a national framework to guide policy development and health system responses to cancer survivorship, focusing on improving the health and wellbeing of people living with and beyond cancer. ${ }^{17}$ National 'optimal care pathways' define recommendations for care across the cancer trajectory, including in the post-treatment follow-up (survivorship) phase, calling for 'screening and assessment of medical and psychosocial 
late effects' and 'interventions to deal with the consequences of cancer and cancer treatments' ${ }^{18}$

Despite these calls for action, care of cancer survivors in Australia continues to focus on cancer surveillance in the specialist setting without sufficiently addressing the concerns or needs of cancer survivors. ${ }^{19}$ With increasing awareness that cancer is a chronic disease for many people, a shift in emphasis is required to a more structured and multidisciplinary approach for prevention and treatment of symptoms, long-term complications and comorbidities to improve survivors' health and wellbeing. To address this, the Clinical Oncology Society of Australia (COSA) Survivorship Group developed a Model of Survivorship Care outlining the critical components of cancer survivorship care with the aim of improving the care of Australians beyond cancer diagnosis and treatment. ${ }^{20}$

\section{Methods}

A multidisciplinary working group of experts from cancer, allied health, primary care and community-based organisations was convened to develop the model using a literature review and consensus processes where evidence was

\section{Box 1. Clinical Oncology Society of Australia position on a model of care for early-stage cancer survivors after completion of primary treatment}

1. Healthcare teams should implement a systematic approach to enhance coordinated and integrated survivorcentred care.

2. Stratified pathways of care are required.

3. Survivorship care should support wellness, healthy lifestyle and primary and secondary prevention while preventing and managing treatmentrelated symptoms, late-term effects and comorbidities, in addition to cancer surveillance.

4. At transition to follow-up care, healthcare teams should develop a treatment summary and survivorship care plan.

5. Survivors require equitable access to services in a timely manner, while minimising unnecessary use of healthcare services and resources. lacking. An iterative development process was undertaken including survey and feedback of COSA members and groups, professional organisations and national consumer advocacy groups.

\section{Recommendations}

The model is outlined in Figure 1. Box 1 summarises the key recommendations.

1. Cancer healthcare teams should adopt a multidisciplinary, systematic approach to deliver coordinated and integrated survivor-centred care for all individuals from the time of diagnosis in partnership with primary care providers. Care teams should support self-management with integration between primary care, community support, non-government organisations and the specialist cancer care services.

Care should be integrated, with recognition that primary care may be best placed to provide preventive care and manage comorbidities, health risk factors (eg smoking, obesity, inactivity) and aspects of cancer follow-up. ${ }^{21}$ Most cancer survivors have comorbid illnesses ${ }^{22,23}$ and existing regular reviews with their general practitioners (GPs).$^{24}$ Integrated care requires clear and timely communication between all healthcare professionals involved in a patient's care. It is critical that professional roles are defined so that all aspects of needed care are provided without duplication.

\section{Stratified pathways of care should be based on the needs and risk factors of the individual, treatment sequelae, existing comorbidities and capacity to self-manage (including assessment of health literacy). Risk of late effects is informed by treatment received. Needs require regular review and update to ensure relevance, with tailored education and rehabilitation as appropriate.}

The COSA model recognises that post-treatment outcomes are informed by events during the diagnostic and treatment phases. Survivorship care should be tailored to the individual's issues, needs and concerns (via a needs assessment such as patient-reported outcome or experience measures) and guided by their risk of developing late and long-term effects, recurrence or a new cancer. ${ }^{12,25}$ Care requirements will also be affected by comorbidities, social circumstances, health literacy and a person's ability and desire to self-manage. Patients who wish to be involved in their own post-treatment cancer care should be provided with the information and support needed to self-manage, as is commonly done for other chronic disease management models (eg diabetes, asthma). ${ }^{21,26}$

\section{In addition to cancer surveillance and management of symptoms and late-term effects, survivorship care should support wellness, healthy lifestyle and primary and secondary prevention.}

Until recently, post-treatment cancer care focused on surveillance for cancer recurrence and/or new primary cancers. ${ }^{2}$ While surveillance remains important, lifestyle factors of survivors increase their risk of developing comorbid illnesses. Many struggle to meet Australian diet and physical activity recommendations. ${ }^{7,27}$ Survivorship care that supports healthy lifestyle behaviours is essential to reduce comorbid disease and maximise recovery and wellness. Referral to allied health professionals and evidence-based programs to assist cancer survivors to be physically active and eat well is critical in achieving these outcomes, as few patients achieve these goals without assistance. Practitioners should refer survivors to local services using chronic disease management GP plans, private health insurance and/or communitybased low-cost programs to engage patients in effective behaviour change. Lifestyle behaviour change programs offer the best available options for long-term prevention, minimisation and management of symptoms and late effects of cancer and its treatments.

\section{At transition to follow-up care, healthcare teams should develop a treatment summary and survivorship care plan as key tools to facilitate comprehensive care planning and communication between healthcare}


providers. The survivorship care plan requires regular review and update to document main concerns and agreed actions.

Transition from intense, acute specialist care to survivorship care can be facilitated by preparing the survivor with timely communication and provision of a treatment summary and survivorship care plan. ${ }^{2,18,20}$ This should include a treatment summary, outlining the cancer diagnosis and treatments, and a plan for follow-up, including strategies to remain well. Survivorship care plans should be completed by a member of the specialist cancer care team with input from the survivor and those involved in providing survivorship care. The care plans should clearly define the role of members in the care team, be regularly reviewed with the GP and other relevant health professionals, be updated as required and be communicated to all parties when changes are made to facilitate communication.

According to a recent systematic review, survivors who received a treatment summary and survivorship care plan reported a greater preference for shared care, were more likely to identify the GP's responsibility in follow-up care, and had more cancer-related contact with their GP and increased implementation of survivorship care recommendations when compared with those who did not receive a treatment summary and survivorship care plan. ${ }^{28}$

\section{Survivors require equitable access to services in a timely manner, while minimising unnecessary use of healthcare services and resources.}

The burden of unmet need and disability after cancer diagnosis varies according to cancer type and treatment, place of residence and socioeconomic status, with patients from rural and remote Australia and lower socioeconomic status reporting more comorbidity, worse health outcomes and less access to cancer care and support. ${ }^{29}$ To ensure care is efficacious and equitable, effective survivorship care needs to be accessible

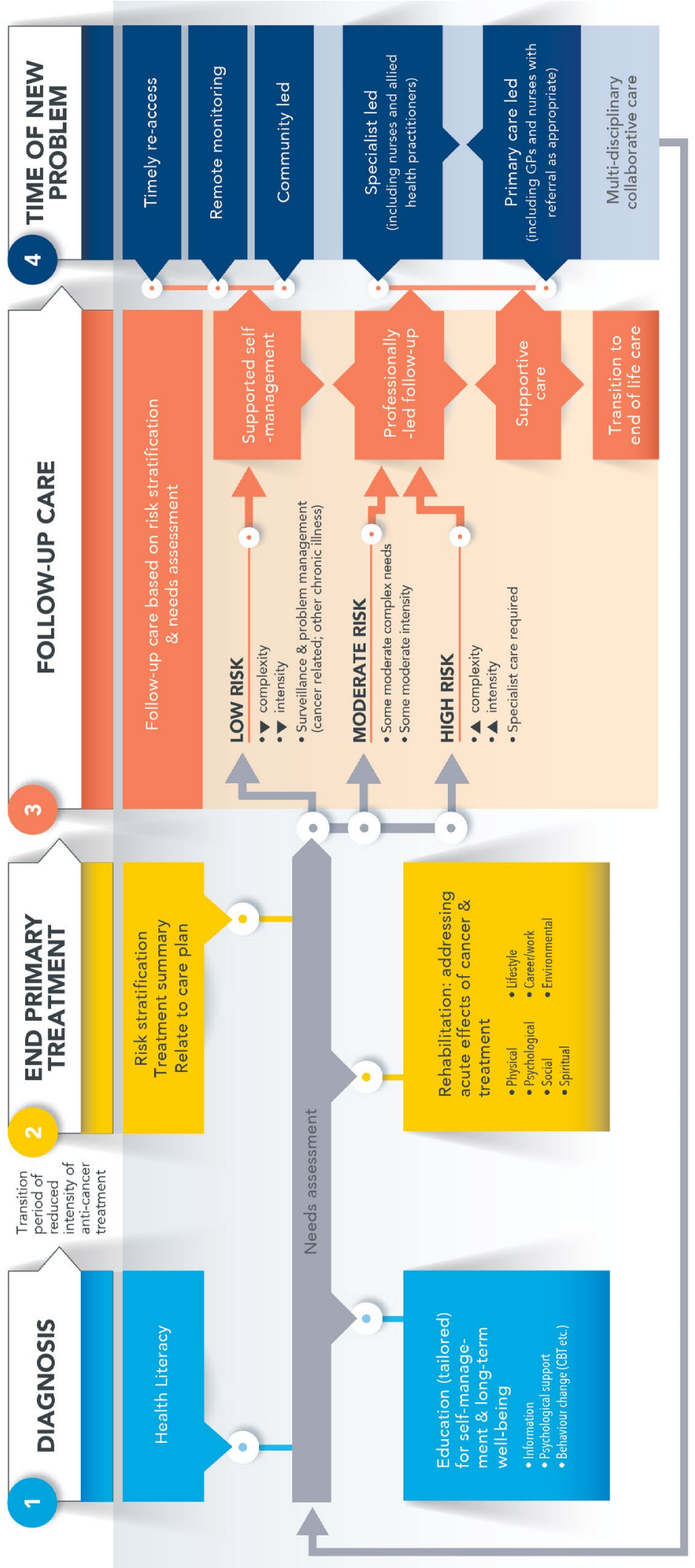

Figure 1. Model of Survivorship Care

Reproduced with permission from the Clinical Oncology Society of Australia 
through novel models of care delivery such as telemedicine and digital technology interventions as well as primary care-led services. An integrated approach that takes advantage of shared care arrangements between cancer services and primary care is likely to be more cost effective. ${ }^{30}$

\section{Conclusions}

An improved model of survivorship care is expected to result in better outcomes for survivors. These outcome measures may include improved duration of survival, reduced risk of cancer recurrence, decreased risk of late effects, better quality of life and improved functional and wellbeing outcomes. At present, the evidence basis remains incomplete, and successful implementation will require research, education, coordination and advocacy. The COSA Survivorship Model of Care provides a template for change, guiding the key steps for implementation into the future.

\section{Authors}

Janette L Vardy BMed (Hons), FRACP, PhD, Professor of Cancer Medicine, University of Sydney, NSW; Director, Sydney Survivorship Centre, NSW; Senior Medical Oncologist, Concord Cancer Centre, NSW. janette.vardy@sydney.edu.au

Raymond J Chan RN, PhD, Professor of Cancer Nursing, Faculty of Health, Queensland University of Technology, Qld; Professor of Cancer Nursing, Division of Cancer Services, Princess Alexandra Hospital, Metro South Health, Qld

Bogda Koczwara AM, BM, BS, FRACP, MBioethics, FAICD, Senior Staff Specialist, Flinders Centre for Innovation in Cancer, Flinders Medical Centre, SA; Senior Staff Specialist, Department of Medical Oncology, Flinders University, SA Karolina Lisy PhD, Senior Research Fellow, Peter MacCallum Cancer Centre, Vic

Richard J Cohn MB, BCh (Rand), DCH SA, FCP (Paed) SA, FRACP, Senior Staff Specialist, Clinical Oncology, Kids Cancer Centre, Sydney Children's Hospital, NSW; Conjoint Professor of Paediatrics, School of Women's and Children's Health, University of New South Wales, NSW

David Joske FRACP, FRCPA, FCHSM, Medical Co-Director, Medical Specialties Division, and Clinical Haematologist, Department of Haematology, Sir Charles Gairdner Hospital, WA Haryana M Dhillon PhD, Senior Research Fellow, Centre for Medical Psychology and Evidence-based Decision-making, University of Sydney, NSW Michael Jefford MBBS, MPH, MHIthServMt, PhD, GCertUniTeach, GAICD, FRACP, Director, Australian Cancer Survivorship Centre - A Richard Pratt Legacy, and Senior Medical Oncologist, Peter MacCallum Cancer Centre, Vic; Professor of Medicine, Sir Peter MacCallum Department of Oncology, University of Melbourne, Vic

Competing interests: MJ reports personal fees in the form of a travel grant from Roche, outside the submitted work. HD reports honoraria paid to her institution from MSD, outside the submitted work. Funding: None.

Provenance and peer review: Not commissioned, externally peer reviewed.

\section{References}

1. Cancer Council Australia. Australians living with and beyond cancer in 2040. Melbourne: Cancer Council Australia, 2018. Available at www.cancervic.org.au/research/registry-statistics/ statistics-data/cancer-prevalence-in-2040.html [Accessed 21 August 2019].

2. Hewitt ME, Ganz PA. American Society of Clinical Oncology (US). From cancer patient to cancer survivor: Lost in transition: An American society of clinical oncology and institute of medicine symposium. Washington: National Academies Press, 2006. p. 189.

3. Baade P, Fritschi L, Eakin E. Non-cancer mortality among people diagnosed with cancer (Australia). Cancer Causes Control 2006;17(3):287-97. doi: 10.1007/s10552-005-0530-0.

4. Holmes MD, Chen WY, Feskanich D, Kroenke $\mathrm{CH}$, Colditz GA. Physical activity and survival after breast cancer diagnosis. JAMA 2005;293(20):2479-86. doi: 10.1001/ jama.293.20.2479.

5. Meyerhardt JA, Giovannucci EL, Holmes MD, et al. Physical activity and survival after colorectal cancer diagnosis. J Clin Oncol 2006;24(22):3527-34 doi: 10.1200/JCO.2006.06.0855.

6. McCabe MS, Bhatia S, Oeffinger KC, et al. American Society of Clinical Oncology statement: Achieving high-quality cancer survivorship care. J Clin Oncol 2013;31(5):631-40. doi: 10.1200/ JCO.2012.46.6854.

7. Tan SY, Turner J, Kerin-Ayres K, et al. Health concerns of cancer survivors after primary anticancer treatment. Support Care Cancer 2019. doi: 10.1007/s00520-019-04664-w.

8. Molassiotis A, Yates P, Li Q, et al. Mapping unmet supportive care needs, quality-of-life perceptions and current symptoms in cancer survivors across the Asia-Pacific region: Results from the International STEP Study. Ann Oncol 2017;28(10):2552-58. doi: 10.1093/annonc/mdx350.

9. Jefford M, Ward AC, Lisy K, et al. Patient-reported outcomes in cancer survivors: A population-wide cross-sectional study. Support Care Cancer 2017;25(10):3171-79. doi: 10.1007/s00520-0173725-5.

10. Koppelmans V, Breteler MM, Boogerd W, Seynaeve C, Schagen SB. Late effects of adjuvant chemotherapy for adult onset non-CNS cancer; cognitive impairment, brain structure and risk of dementia. Crit Rev Oncol Hematol 2013;88(1):87-101. doi: 10.1016/j.critrevonc.2013.04.002.

11. de Boer AG, Taskila T, Ojajärvi A, van Dijk FJ, Verbeek JH. Cancer survivors and unemployment: A meta-analysis and meta-regression. JAMA 2009;301(7):753-62. doi: 10.1001/jama.2009.187.

12. Shapiro CL. Cancer Survivorship. N Engl J Med 2018;379(25):2438-50. doi: 10.1056/ NEJMra1712502.

13. Cancer Australia. Recommendations for the identification and management of fear of cancer recurrence in adult cancer survivors. Surry Hills, NSW: Cancer Australia, 2014.

14. Lobb EA, Joske D, Butow $P$, et al. When the safety net of treatment has been removed: Patients unmet needs at the completion of treatment for haematological malignancies. Patient Educ Couns 2009;77(1):103-08. doi: 10.1016/j.pec.2009.02.005

15. Boyes AW, Girgis A, Zucca AC, Lecathelinais C. Anxiety and depression among long-term survivors of cancer in Australia: Results of a population-based survey. Med J Aust 2009;190(7 Suppl):S94-98. doi: 10.5694/j.13265377.2009.tb02479.x

16. Hewitt M, Greenfield S, Stovall E. From Cancer patient to cancer survivor - Lost in transition: An American society of clinical oncology and institute of medicine symposium by Institute of Medicine. Washington, DC: National Academies Press, 2006.

17. Cancer Australia. Principles of cancer survivorship. Canberra: Cancer Australia, 2017.

18. Cancer Council Victoria. Optimal care pathways. Melbourne: Cancer Council Victoria, 2019. Available at www.cancervic.org.au/for-healthprofessionals/optimal-care-pathways [Accessed 21 August 2019].

19. Lisy K, Langdon L, Piper A, Jefford M. Identifying the most prevalent unmet needs of cancer survivors in Australia: A systematic review. Asia Pac J Clin Oncol 2019;15(5):e68-e78. doi: 10.1111/ ajco.13176.

20. Clinical Oncology Society of Australia Model of Survivorship Care Working Group. Model of survivorship care: Critical components of cancer survivorship care in Australia position statement. Sydney, NSW: COSA, 2016

21. Rubin G, Berendsen A, Crawford SM, et al. The expanding role of primary care in cancer control. Lancet Oncol 2015;16(12):1231-72. doi: 10.1016/S1470-2045(15)00205-3.

22. Bluethmann $S M$, Mariotto $A B$, Rowland $J H$. Anticipating the 'silver tsunami': Prevalence trajectories and comorbidity burden among older cancer survivors in the United States. Cancer Epidemiol Biomarkers Prev 2016;25(7):1029-36. doi: 10.1158/1055-9965.EPI-16-0133.

23. Berry NM, Miller MD, Woodman RJ, et al. Differences in chronic conditions and lifestyle behaviour between people with a history of cancer and matched controls. Med J Aust 2014;201(2):96-100. doi: 10.5694/mja13.10701.

24. Heins MJ, Korevaar JC, Donker GA, Rijken PM, Schellevis FG. The combined effect of cancer and chronic diseases on general practitioner consultation rates. Cancer Epidemiol 2015;39(1):109-14. doi: 10.1016/j. canep.2014.12.002.

25. Alfano CM, Mayer DK, Bhatia S, et al. Implementing personalized pathways for cancer follow-up care in the United States: Proceedings from an American Cancer Society - American Society of Clinical Oncology summit. CA Cancer J Clin 2019;69(3):234-47. doi: 10.3322/caac.21558.

26. Smith SM, Allwright S, O'Dowd T. Effectiveness of shared care across the interface between primary and specialty care in chronic disease management. Cochrane Database Syst Rev 2007;3:CD004910. doi: 10.1002/14651858. CD004910.pub2.

27. Eakin EG, Youlden DR, Baade PD, et al. Health behaviors of cancer survivors: Data from an Australian population-based survey. Cancer Causes Control 2007;18(8):881-94. doi: 10.1007/ s10552-007-9033-5.

28. Jacobsen PB, DeRosa AP, Henderson TO, et al. Systematic review of the impact of cancer survivorship care plans on health outcomes and health care delivery. J Clin Oncol 2018;36(20):2088-100. doi: 10.1200/ JCO.2018.77.7482.

29. Fox P, Boyce A. Cancer health inequality persists in regional and remote Australia. Med J Aust 2014;201(8):445-46. doi: 10.5694/mja14.01217.

30. Emery JD, Jefford M, King M, et al. ProCare Trial: A phase II randomized controlled trial of shared care for follow-up of men with prostate cancer. BJU Int 2017;119(3):381-89. doi: 10.1111/bju.13593. 